\title{
Personal Health Monitoring with Mobile Application Based on Blockchain Technologies
}

\author{
Nataliya Apatova*, Oleg Korolyov, and Sergey Ivanov \\ V.I. Vernadsky Crimean Federal University, 4, Vernadsky av., Simferopol, Russia
}

\begin{abstract}
Personal health monitoring is especially necessary in a pandemic of COVID19 and based on objective and subjective data. Modern medicine uses numerous diagnostic devices, many of which are for personal health monitoring. Applications for mobile phones are becoming more widespread, they make a possibility constantly monitor vital signs for a person. However, the consolidation into a single personalized database of information on daily mobile monitoring and examination results from various doctors in various medical organizations not yet carried out. Proposed to build a blockchain from this data and results of data analysis add subjective sensations and indicators to it, which clarified during the conversation with the doctor and not always fully and correctly transmitted by the patient. Using an integrated approach to personal health monitoring, building a blockchain from official data and personal objective and subjective indicators makes it possible to identify at the early stages of the disease, to have a complete and reliable picture of the state of health.
\end{abstract}

\section{Introduction}

Modern healthcare operates on four principles called "4P": prediction, prevention, partnership and personalization [8].

Each person has individual characteristics of his body, determined by heredity, lifestyle, profession and environment. In this regard, the medical examination carried out by the state, medical examinations annually or once every several years, make it possible to implement the first principle and predict possible diseases, prevent them or reduce risks for a person.

In medicine, the prevention of various diseases has always been considered important, which requires much lower costs than subsequent treatment and the lost benefit from the absence of an employee from his workplace. As shown by O.A. Antipova and E.V. Ogloblina, in 2016 the economic damage from cardiovascular diseases in Russia amounted to 2.7 trillion. Rubles or $3.2 \%$ of GDP, "which is comparable to the expenditures of the RF budget for health care" [1, p. 252]. Prevention implements the second principle - disease prevention.

\footnotetext{
*Corresponding author: apatova@list.ru
} 
The third principle related to the implementation of the partnership between doctors and the patient, since the patient is a participant in the treatment process and it is necessary that he observe all the doctor's prescriptions.

A separate area of modern healthcare is personalized, individual medicine, which also offers opportunities for using digital technologies. The ecosystem of personalized health care includes the patient himself and related services and groups: the MHIF system (compulsory health insurance fund), social insurance fund, insurance companies, employer, pharmacies, relatives, sanatoriums, doctors, clinics, emergency medical care and hospital. Monitoring the health of every citizen and its remote implementation intended primarily for people suffering from chronic diseases, because according to statistics, from 20 to $30 \%$ of such citizens cannot visit the clinic on their own. Remote monitoring combines the capabilities of artificial intelligence systems, the Internet of Things with video and conferencing. So "expert data based on the results of international projects predict the following application results: 1) a $35-56 \%$ decrease in mortality; 2) up to $47 \%$ reduction in the likelihood of hospitalization; 3 ) up to $65 \%$ reduction in the number of visits to the clinic; 4) a $63 \%$ reduction in transportation costs " [1, p. 255].

The most promising, in our opinion, will be personalized dynamic databases in the form of a block chain and containing information from various medical organizations visited by the patient, his personal objective data obtained as a result of the operation of mobile applications, as well as observations of his own state of health, which have the form accumulated messages.

\section{IT trends and results in Russian medical sphere}

To ensure measures for the digitalization of healthcare in Russia, a number of resolutions adopted. They include follow documents. First, Government Decree "On the Unified State Information System in the Healthcare Sector" (No. 555 of May 5, 2018). Second, The Order of the Ministry of Health of Russia "On approval of the procedure for the organization and provision of medical care using telemedicine technologies " (No. 965n of November 30, 2017); third, Government Decree on the interaction of other information systems in the field of health care, the relevant bylaws (No. 447 of April 14, 2017 (last edition)). These documents govern the rules for the development and interaction of various information systems in the healthcare sector, the organization of storage and access to data, the organization of the use of information technologies in clinical processes, etc.

In 2018, the Digital Healthcare Consortium created in the Russian Federation, in addition to which the Unified State Information System in the Healthcare Sector (YHISZ) and the Unified Medical Information and Analytical System of Moscow (UMIAS) are already operating. "The goal of the Consortium is to prepare the transfer of all national medicine to the model of a" digital twin "of each person, no matter in what remote settlement he is" [2, p. 5].

Many hospitals, private and public, use electronic health records (EHRs), which store complete patient records. However, the use of EHR still does not allow patients to choose freely a clinic and a specialist, since information from the card not transferred outside the hospital. This is a big disadvantage - if a person seeks medical help elsewhere, he has to undergo diagnostics again. In 83 regions of the Russian Federation, EMH of 46 million patients carried out; there is also the possibility of electronic appointment with a doctor. On the portal of State services there is a personal account "My health", the services "Providing information about attachment to a medical organization", "calling a doctor at home" work.

Telemedicine should become one of the leading technologies in the field of healthcare. It facilitating medical examination at a distance. By 2025 , at least $55 \%$ of medical institutions in Russia must implement telemedicine technology to serve patients - this is the task of the 
priority project "E-Health", which is being implemented by Royal Philips (Netherlands). It is one of the leading technology companies in solving problems of improving the quality of life; from leading a healthy lifestyle, prevention and early diagnosis, to treatment and home care. In Russia, portable ultrasound devices of this company are available, which are compatible with smartphones or tablets. The Philips' Future Health Index 2018 study showed that $82 \%$ of the Russian population would like to consult a doctor remotely in certain life situations. The dissemination of telemedicine solutions hampered by patients' concerns about the safety of their personal information. In 2019, a study showed that $34 \%$ of those residents of Russia who currently do not use digital medical technologies said that they begin to do when they are confident in the confidentiality of their data.

Telemedicine makes it possible, for example, to transfer X-rays and images obtained a result of CT or MRI examinations to medical centers thousands of kilometers away from the place of care in a situation where there is no specialist or additional expert opinion and need to be sure of a conclusion. These data displayed on the computer and the doctor remotely analyzes the information received and clarifies the diagnosis.

For patients, the possibilities of digitalizing healthcare are follows. First, the availability of medical care will increase due to the more efficient organization of the resources available in the health care system, especially in high-tech medical care. Secondly, the use of telemedicine technologies provided the required level of accessibility of narrow specialists, including for residents of rural areas and small settlements. Thirdly, medical documentation convert into electronic form and patients have access to it through the My Health office on the Unified Portal of Public Services. Fourthly, unnecessary bureaucratic links associated with the significant time spent by people on paperwork and organizing access to medical services remove by introducing electronic document management and transferring most of the services to electronic form. Fifthly, most importantly, the quality of the medical care provided will increase due to the latest diagnostic and treatment methods, systems for interpreting the results of medical research based on information technologies, which lead to a reduction in the number of medical errors, a decrease in the waiting time for medical care, and an increase in the effectiveness of treatment.

Access to medical information about a patient provided for doctors regardless of the place and time of medical care, which ensure the continuity of patient treatment. A doctor receives decision support systems based on artificial intelligence technologies, other innovative developments in the field of diagnostics and treatment. He has the ability to carry out remote consultations with colleagues, make collective decisions, continuously study online and improve his skills.

The digitalization of healthcare not limited to the introduction of electronic health records and remote consultations. Technologies for working with big data have enormous potential, and medical technology companies are trying to unleash it as much as possible. The Philips Research Laboratory in Skolkovo develops new high-tech medical solutions, among which there are computer vision algorithms that can analyze and interpret information in images. With its help, doctors will be able to perform CT and MR diagnostics much faster and more accurately.

Recently, especially in connection with the coronavirus pandemic, the media have been discussing the issues of chipping people, which means "the process of implanting a special electronic mini-device with an information code under the human skin. The information in it is intended for reading by special scanners created for this very purpose" [3, p. 22]. At present, chips implanted only in animals, and for humans they are outside the legal field, there are no laws about this. For medicine, such a chip can help provide a person with quick help, diagnose the cause of a worsening condition. However, "With the help of the RMS computer, the operator can send electromagnetic signals to the nervous system, thus affecting the person. Electromagnetic stimulation can interfere with brain function and 
muscle activity, causing spasms and bouts of severe pain, similar to torture. In this case, the inviolability of the person implanted with the chip violated for life. ... The chip can be controlled not only in a positive direction of development, but also in a negative direction, up to the reduction of the country's population" [3, p. 23]. Thus, telemedicine can use not for the benefit of people, but against them.

The following IT technologies used in medicine: Internet of Things services; block chain; artificial intelligence and contextual recognition algorithms; robots: adaptive, intelligent and imitation of presence [4].

The implementation of the Digital Economy in Healthcare Project in Russia consists of four stages:

1) Assessment of the prospects for sustainable economic development;

2) Effective implementation of the national project "Health";

3) Program for the development of innovative technologies in medicine;

4) Improving the quality of medical services provided.

For the successful implementation of the listed stages, it is necessary to develop or improve the following information systems:

1) Portal for management of innovative technologies in medicine;

2) Portal of information and communication technologies in medicine:

3) Portal for managing economic and legal risks of telemedicine.

The process of digitalization of healthcare is global; to one degree or another it has developed in different countries. Since about the mid-2000s the global transition to digital technologies gave impetus to the development of an interdisciplinary field of scientific knowledge - medical informatics the development of which is at a faster pace than the development of both medicine and computer science separately. The development of digital health care and medicine in itself is unlikely at the present to have a strong influence on their effectiveness in terms of average life expectancy, with a clear positive effect in a purely economic respect in the organization of health care. Russia underutilizes the existing potential for the development of digital technologies and the level of staffing in medicine $[5]$.

The development of digital technologies related to improving the health of the population can be a catalyst for the activation of social entrepreneurship in this area. We can talk about social entrepreneurs who develop innovative solutions using digital technologies that can become part of medical activities or the development of medical services. In particular, it can be mobile applications for notifying patients about upcoming procedures, information and educational portals on the prevention and treatment of diseases. This also includes the development of Internet of Things technologies that allow overcoming, compensating or replacing life limitations, the development of software and hardware systems for the rehabilitation of disabled people, the improvement of telemedicine technologies, etc. [6].

\section{Personal medical digital data}

The concept of a health electronic messaging system in the United States defined as "an electronic application by which people can access, manage and share their health information in a private, secure and confidential environment" [7].

Possible functions of electronic health records are the following.

1. Access to the provider's electronic medical history (short or detailed); for example, medical history, medications and tests.

2. Personal organizer or health diary - for example, clinics, doctors, tests, visits, overthe-counter treatments, scanned documents. 
3. Support for self-management included care plans, graphical displays of symptoms, passive biofeedback, individualized instructional or motivational feedback, decisionmaking or reminders

4. Secure communication between patient and healthcare provider to make appointments, reorder prescriptions, or receive advice (patient and doctor email)

5. Links to static or interactive information about disease, treatment or self-service

6. Links to sources of support such as patients or virtual peer networks.

7. Collect data on symptoms or health behavior through self-report or objective monitoring using mobile devices (stationary or portable)

In Europe, there is also the concept of electronic health records, which mainly focus on accessible data access [8]. Following promising functions of electronic health records highlight:

- Access to the electronic medical history (short or detailed), for example, to using medications and test results.

- Personal organizer or health diary - for example, clinics, doctors, tests, visits, overthe-counter treatments, scanned documents.

- Self-monitoring support using care plans, graphical displays of symptoms, passive biofeedback, personalized instructional or personalized feedback, decision aids or reminders.

- Secure communication between patient and healthcare provider to make appointments, reorder prescriptions, or receive advice (with patient and doctor email).

- Links to static or interactive information about illness, treatment, or self-care.

- Links to sources of support such as patients or virtual peer networks.

- Collect data on symptoms or health status through self-report or objective monitoring using electronic devices (stationary or portable).

Patient health care provider email and remote symptom submission to electronic health records also tested in both sectors.

The benefits of using personal electronic health records highlighted. First, Electronic Personal Health Records can empower patients through greater access to personal data, health information, and communication tools that can aid in self-care, collaborative decision-making and clinical outcomes. Second, they can also reduce geographic barriers to patient care and act as an integration point for records, especially in fragmented health systems, thereby increasing the continuity and efficiency of care. $[9,10]$ Third, the quantity and quality of research remains limited, existing research suggests improved communication and trust between patients and professionals, confidence in self-care, adherence to chronic conditions, and accuracy of records [11, 12].

C. Pagliari and D. Detmer identify the following factors determine the adaptation and efficiency of the of electronic health records using. First, confidentiality and security of data use. Second, the digital divide which means unequal opportunities in physical access and use of digital infrastructure - lack of access to the Internet, computer or mobile phone, and the lack of the necessary minimum digital competencies at the level of basic technical skills in using the digital environment. Third, the choice of a model which involves finding a compromise between the complexity, heterogeneity of the digital environment, coordination of various participants and accessibility, control over personal data and the security of the formation and use of personal medical records. Forth, technical aspects of implementation, human and organizational factors. 


\section{Blockchain technology for subjective and objective personal health data}

The gradual development and improvement of the idea proposed by Nakamoto led to the fact that the blockchain stated as a separate technology [13]. Currently, block chain technology, together with cryptocurrencies, already considered as fully developed and solid technologies. There are more and more modern high-tech applications created using block chain technology.

The authors of the article are confident that the use of blockchain technology is appropriate when developing a personal health monitoring system. Next, we will give a number of facts and statements that allow us to show the positive aspects and benefits of using blockchain in a system related to the collection of personal health data, as well as storing this data in compliance with confidentiality conditions and guaranteeing the integrity of this data. The most traditional and commonly used application of blockchain technology has been cryptocurrencies and electronic payment systems. That is why that the following main arguments and examples are from these areas.

A network of interacting agents performs data state coordination and synchronization when using blockchain technology. Most often, such synchronization performed at regular intervals; however, different variations are also possible in this matter. A huge advantage of the technology is that it does not impose any restrictions on the type or kind of stored data. This property makes the technology extremely versatile and allows distributed databases to track changes and establish an exchange between different types of digital data [14]. The rules by which the network reaches consensus on the state of the shared data and maintains this consensus over time are the key aspect in the use of this technology.

Such a distributed base, which contains the general data of the system, called blockchain. It is usually a chain of blocks of transaction data. Each of the blocks contains the actual records of transactions performed over a certain period, as well as additional attributes of these transactions.

It is obviously that personal health data is highly sensitive with respect to reliability and integrity. Any unauthorized access to data and their modification can lead to an incorrect diagnosis, and this, in turn, can have tragic consequences for a person. Therefore, the issues of system security and data integrity are one of the key issues in the design and development of personal health monitoring systems.

The information stored in the database about the completed transactions can contain an arbitrary number of attributes. The key attribute for each transaction and each block in the chain is the timestamp. Blocks formed that connected together when building a chain. They necessarily include the digital signature (hash) of the previous block in the current block. Such a structure is necessary because any change made to the information about a transaction contained in this particular block will necessarily change such a signature. The change becomes impossible since it will necessarily break the chain of consensus that links this block with all subsequent ones.

The specified feature of blockchain technology allows us to talk about a sufficient degree of reliability of personal data storage. This fact gives us the right to assert that the use of blockchain technology in the personal health monitoring system is a guarantee of the integrity of personal data.

Thus, we can use blockchain technology not only as a basis for developing a large distributed database, but also as a control tool. The specified structure of the chain, when each of its blocks includes information about all subsequent blocks of the chain, makes it completely impossible to change the data so that it not noticed.

Despite the high degree of development of modern technologies, in the development of distributed information storage systems, maintaining the relevance and integrity of such a 
system is still expensive. The concept of a high price includes the cost of using various technologies, attracting highly qualified specialists and purchasing expensive equipment. At the same time, to ensure the integrity of transactions, it may require not only one or more trusted intermediaries, but also the involvement of several parties to agree on the rules for secure data entry and sharing.

From the above, we can conclude that the use of blockchain technology not only guarantees the reliability of transactions, but also reduces the cost of maintaining such a system by eliminating the services of intermediaries.

A feature for transactions that carried out in networks using blockchain technology is that the cost of such transactions within the network is practically zero [15]. Moreover, such a low cost of transactions does not depend on the amount involved in the transaction at all.

Note that such transactions confirmed within a few seconds or at most a few minutes. The speed of transaction confirmation does not depend on the distance between the sender and the recipient. Execution of the operation completely regardless of the jurisdiction of residence of the actors who initiated the execution of the transaction. Thus, unlike traditional payment infrastructure, which supports, for example, credit cards, money transfers, or international bank transfers, digital currencies not bound by the rules or legal status of any one nation's currency. This feature allows significant freedom in performing operations and significantly reduces overhead costs.

This feature of blockchain technology can also considered exclusively as a positive quality of the technology and an additional argument in favor of using this technology in the personal health monitoring system.

Note that blockchain technology can see as a means of preventing information leakage. At the same time, it provides all system participants with the opportunity to check transaction attributes and conclude contracts. The advantage of using technology in favor of enhancing security is that the information in the system not provided to a third party to confirm its truth. The technology itself allows the agent to check any piece of information in the system and make sure it is correct without giving full access to all the original information. That is, the technology allows checking the attributes of transactions in privacy mode.

The use of digital technology has led to the fact that for many types of transactions their cost has decreased. Moreover, the decline was quite significant and in some cases led to almost the disappearance of value. Blockchain technology completes this process, avoiding unnecessary checks. In particular, blockchain technology such as that used in bitcoin can used for cheaply verify ownership and exchange in cryptocurrency.

While the bitcoin network consumes computing power to support transactions and expand its distributed ledger, it is important to emphasize that such energy consumption is low compared to the labor and capital costs involved in enabling transactions in traditional financial infrastructure [16]. In addition, while existing intermediary payment platforms have access to all transaction data and accumulate bargaining power, blockchain technology promotes the exact opposite approach, as low barriers to entry and innovation provide a higher level of competition for different types of services.

What also distinguishes blockchain-based payment methods from many others, such as PayPal or credit cards, or even bank transfers, is the irreversibility of transactions [17]. This means that once the transaction is completed, there is no way to undo it. Instead, a new transaction created, which should actually do the opposite. In the case of payment systems, this feature is controversial. On the one hand, irreversibility can be a good feature when viewed from the perspective of a seller who expects his service or product unambiguously paid for. On the other hand, when viewed from the buyer's perspective, irreversibility considered a bad feature because the purchase can be unwanted or erroneous. While buyers 
and sellers can always voluntarily agree to bug fixes, in general, blockchain-based payment networks like Bitcoin do not have built-in mechanisms for fixing bugs. In addition, this accumulation of transactions leads to increase the amount of stored data.

However, in the case of a personal health monitoring system, this feature is an advantage. If measurements made with the help of a certain device, then they must entered into the system. At the same time, if the obtained measurements were doubtful, then the person performs repeated measurements and they entered into the system as new results and do not override the information received recently.

Another feature is that blockchain technology allows a network of interacting agents to agree on the true state of shared data at regular intervals. This feature allows automatically solve the problem of synchronization and updating of distributed data. It is not a secret that this task is quite laborious and, with large amounts of data, requires special attention and special technical means and approaches. The use of blockchain technology allows you to solve this problem without resorting to additional steps.

Blockchain technology can also attributed to the fact that it is widely used in distributed and secure data repositories. Obviously, the technology itself is only part of what can used in the development of secure repositories. At the same time, using decentralized, encrypted communication protocols together with blockchain technology, we get not only secure ways to store sensitive data, but also the ability to send and receive messages without the risk of interference.

The unique features of blockchain technology will simultaneously significantly secure confidential data and unambiguously and accurately identify their owner.

The potential patient collects his health information in the form of messages that come in as unpleasant symptoms, such as headaches, high blood pressure. These symptoms cannot measured with common mobile applications, but they can handled with software that tracks the frequency and severity of symptoms, such as severe or mild headaches. Messages accumulated in the database of the application and, with a certain "critical mass", can form a feedback in the form of a recommendation to examine by a doctor. A person (or a doctor) can independently request information about objective data, which represent the results of previous examinations and measurements in different medical institutions. These results and subjective information from the database of personal messages will be the blockchain, the sequential viewing of which will make it possible to identify the patient and obtain a complete picture of his physical condition and history of the disease.

\section{Conclusion}

The digitalization of healthcare is providing great new opportunities for patients and doctors. Firstly, these are electronic medical records, which already used in many countries and allow storing information about a patient's health in various forms and databases. The disadvantage is the presence of an electronic medical record in one institution and the use of software, which is not yet perfect, to communicate data from different organizations. Secondly, telemedicine makes it possible to use experts for medical consultations for complex cases that are geographically remote from the expert. Government programs aimed at solving organizational problems in health care, but the problems of collecting personal health data and the ability to collect all information quickly from different sources remain unresolved. Thirdly, information about the patient's health reduced to the data of objective measurements and analyzes, but does not take into account his personal observations and may miss important symptoms of ailments that the patient describes in text or oral message. 
Using an integrated approach to personal health monitoring, building a block chain from official data and personal objective and subjective indicators makes it possible to identify at the early stages of the disease, to have a complete and reliable picture of the state of health.

Blockchain technologies for personal health monitoring have the following advantages. First, they allow quickly and with low financial costs combine health data into one chain, which provides a doctor or patient with comprehensive information about his health status. Secondly, this data is confidential and reliably protected by the technology itself. Thirdly, in addition to data from official sources, personal observations enter into the chain in the form of messages and patient complaints, they can tracked over time and cannot changed, which allows get a complete picture of the health status and make the right decision about treatment.

In further studies, we plan to improve a mobile application with artificial intelligence elements for storing and classifying oral complaints of a patient before going to a doctor and to create a pilot blockchain monitoring project

\section{References}

1. O.A. Antipova, E.V. Ogloblina. The use of promising digital technologies in health care is a necessary condition for increasing the competitiveness of the Russian economy // Scientific works of the free economic society of Russia..Vol. 217. No. 3. (2019).

2. O.A. Golodova, N.R. Mamutov. Trends in the development of digital health care in the Russian Federation // Actual problems of modern economy. No. 4. (2019.).

3. L.V. Semenova, V.A. Perminova. Research on chipization in the digital economy and healthcare // Science of Europe. No. 41-2 (41).(2019).

4. P. Rolinson, E.A. Arievich., D.E. Ermilova. Objects of intellectual property created using artificial intelligence: features of legal the regime of Russia and abroad // Law. No. 5.(2018).

5. Yu.A. Morozova. Digitalization as a global, country and sectoral process in improving the effectiveness and efficiency of healthcare and medicine // Intellect. Innovation. Investments.No. 4. (2019).

6. E.V. Rozhkova. Ensuring the availability of preventive medical services: the possibilities of digital technologies // Intellect. Innovation. Investments.No. 7. (2019).

7. Connecting for Health. Connecting Americans to their healthcare. Final report of the working group on policies for electronic information sharing between doctors and patients New York: Markle Foundation.

www.connectingforhealth.org/resources/final_phwg_report1.pdf (2004).

8. C. Pagliari, D. Detmer, P. Singleton P. Potential of electronic personal health records. BMJ.;335 (7615). (2007).

9. PC. Tang, JS Ash, DW Bates, JM Overhage, DZ Sands. Personal health records: definitions, benefits, and strategies for overcoming barriers to adoption. J Am Med Inform Assoc. 13:121-6.( 2006).

10. N. Richards. My illness, my record. BMJ. (2007).

11. C. Pyper, J. Amery, M. Watson, C. Crook. Patients' experiences when accessing their on-line electronic patient records in primary care. Br J Gen Pract. 54. (2004).

12. A. Honeyman, B. Cox, B. Fisher. Potential impacts of patients' access to their electronic records. Inform Primary Care, 13. (2005). 
13. Nakamoto S. A Peer-to-Peer Electronic Cash System: https://bitcoin.org/bitcoin.pdf

14. Ledger Technology: beyond block chain. A report by the UK Government Chief Scientific Adviser / Government Office for Science. (2016).

15. Bitcoin Wiki. Transaction Fees. (https://en.bitcoin.it/wiki/ Transaction_fees).

16. EBA. Warning to Consumers on Virtual Currencies. European Banking Authority, EBA/WRG/2013/01.

17. Davidson, S., P. De Filippi, and J. Potts: Economics of blockchain,". Working Paper (2016). 